\title{
Evaluation of the clinical significance of human papillomavirus (HPV) 53
}

\author{
Elizaveta Padalko ${ }^{\mathrm{a}, \mathrm{d}}$, Catherine Ali-Risasi ${ }^{\mathrm{b}}$, Lieve Van Renterghem ${ }^{\mathrm{a}}$, Mieke Bamelis ${ }^{\mathrm{b}}$, \\ Anja De Mey ${ }^{\mathrm{b}}$, Yolande Sturtewagen ${ }^{\mathrm{b}}$, Hilde Vastenavond ${ }^{\mathrm{b}}$, Davy Vanden Broeck ${ }^{\mathrm{c}, *}$, \\ Steven Weyers ${ }^{\mathrm{c}}$, Marleen Praet ${ }^{\mathrm{b}}$
}

\footnotetext{
${ }^{a}$ Department of Clinical Chemistry, Microbiology and Immunology, Ghent University Hospital, De Pintelaan 185, 9000 Ghent, Belgium

b Department of Pathology, Ghent University Hospital, De Pintelaan 185, 9000 Ghent, Belgium

' Department of Gynecology, Ghent University Hospital, De Pintelaan 185, 9000 Ghent, Belgium

d School of Life Sciences, Hasselt University, Agoralaan Building D, 3590 Diepenbeek, Belgium
}

\section{A R T I C L E I N F O}

\section{Article history:}

Received 9 September 2014

Received in revised form 11 March 2015

Accepted 8 April 2015

\section{Keywords:}

Human papillomavirus

Possible high-risk type

Cervical cancer

HPV 53

\begin{abstract}
A B S T R A C T
Objective: Human papillomaviruses (HPV) are classified according to their potential for the development of cervical neoplasia. However, the carcinogenicity of HPV types forms an evolving continuum based on the newly available data especially regarding the role of probable and possible high-risk HPV types (pHRHPV). The objective of the present work was to evaluate clinical significance of the pHR-HPV53.

Study design: An observational cohort study of potential aetiological association between infection with HPV53 and development of high-grade cervical cytology was performed. The study was conducted in two geographically remoted hospitals, in Belgium and Democratic Republic of Congo, as an attempt to collect data from regions with different geographical distribution of HPV genotypes. The samples were taken during routine gynaecological visit in outpatient clinics of both participating hospitals.

Results: A total of 2283 liquid-Pap samples were taken from 1465 women at Ghent University Hospital, Belgium, and from 660 women at General Hospital and Ngaliema Hospital of Kinshasa, DRC. "HPV53only"-pattern as evaluated by full HPV genotyping was found in samples from only 34 (1.6\%) samples. The initial cytology represented next to non-dysplastic, undetermined and low-grade lesions also highgrade lesions (12\%). For 26 (76.5\%) from the 34 women presented with "HPV53-only"-pattern follow-up results were available showing no progression to malignancy.

Conclusion: Our findings support very low to lacking carcinogenic potential of HPV53. Recognising extreme rarity in cervical cancer next to high prevalence in general population of HPV53, further studies investigating progression to high-grade lesions are needed to elucidate the oncogenic potential of pHRHPV53.
\end{abstract}

(c) 2015 Published by Elsevier Ireland Ltd.

\section{Introduction}

Epidemiological classification of human papillomaviruses (HPV) as high, low and probable high-risk is based on their association with cervical cancer. HPV types 26, 53 and 66 are within this classification determined as probable high-risk types based on their detection in few malignant cases and only in controls $[1,2]$. According to the recent review of International Agency for Research on Cancer (IARC) assessing carcinogenicity of biological

\footnotetext{
* Corresponding author at: International Centre for Reproductive Health, Ghent University Hospital, De Pintelaan 185, 6K4, B-9000 Ghent, Belgium. Tel.: +32 9 3323564; fax: +32 93323867 .

E-mail address: davy.vandenbroeck@ugent.be (D. Vanden Broeck).
}

agents, HPV types $26,53,66,67,70,73$ and 82 as remaining types in mucosotropic alpha genus are classified as "possibly carcinogenic" based on limited evidence of their involvement in cervical cancer while HPV68 is classified as "probably carcinogenic" acknowledging its strong mechanistic evidence [3]. Recent studies have shown viral oncogene transcription of possible high-risk HPV (pHR-HPV) types $26,66,70$ and 82 in cervical cancer as well as detection of HPV73 in cancer specimens highlighting oncogenic potential of these pHRHPV types and supporting reassessment of their carcinogenicity classification while HPV53 was shown to be lacking biological activity in cervical cancer $[4,5]$.

Especially differences in carcinogenic potential between HPV53 and HPV66 are intriguing as they both belong phylogenetically to $\alpha-6$ species, show almost similar prevalence in low-grade squamous intraepithelial lesions (LSIL) as well as an comparable 
Table 1

Initial cytological results of "HPV 53-only" positive women.

\begin{tabular}{|c|c|c|c|c|c|}
\hline Hospital & “HPV 53-only” & NILM & ASC-US & LSIL & HSIL \\
\hline Ghent University Hospital, Belgium & 33 & 2 & 8 & 19 & 4 \\
\hline General Hospital and Ngaliema Hospital of Kinshasa, DRC & 1 & & 1 & & \\
\hline
\end{tabular}

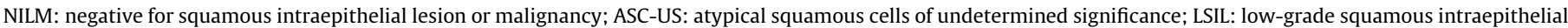
lesion; HSIL: high-grade squamous intraepithelial lesion.

estimated potential for progression to malignancy and are both highly genetically diverse with distinct intratype variants [6-9]. There is currently no evidence that L1 polymorphisms are associated with cervical malignancy but the diversity of these types and their worldwide distribution are striking [10-13]. While the detection of HPV66 is currently included in the majority of commercially available tests for cervical cancer screening, convincing data on the clinical significance and malignant potential of HPV53 are lacking $[14,15]$.

The objective of the present work was to evaluate clinical significance of the HPV53 on the follow-up data of cervical smears initially positive for HPV53 and not accompanied by presence of any tested high-risk HPV type ("HPV53-only").

\section{Materials and methods}

An observational cohort study of potential aetiological association between infection with HPV53 and development of highgrade cervical cytology was performed. The study was conducted in geographically remoted hospitals, Ghent University Hospital, Belgium and General Hospital and Ngaliema Hospital of Kinshasa, Democratic Republic of Congo (DRC), as an attempt to collect data from regions with different geographical distribution of HPV genotypes. Recruitment in Ghent University Hospital took place from February 2008 till July 2009 and in General Hospital and Ngaliema Hospital of Kinshasa from July 2009 till August 2009. The samples were taken during routine gynaecological visit in outpatient clinics of both participating hospitals. The samples were referred for HPV testing after an initial cytological screening of the liquid-based Pap test (PreserCyt Solution; Cytoc UK, Crawley, UK). The evaluation of cervical lesions was performed according to Bethesda classification of cervical cytology: Negative for squamous Intraepithelial lesion or malignancy (NILM), Atypical Squamous Cells of Undetermined Significance (ASC-US), Low-grade Squamous Intraepithelial Lesion (LSIL) and High-grade Squamous Intraepithelial Lesion (HSIL). HPV DNA detection was performed using the HPV Full Spectrum PCR Amplification and Detection/ Genotyping System (Lab2Lab Diagnostic Services; GenoID Molecular Diagnostics Laboratory, Budapest, Hungary), recognising HPV types $16,18,31,33,35,39,45,51,52,56,58,59,66,68,6,11,42$, 43,44 and 53 [16]. The subject was considered to be eligible for the study if "HPV53-only"-pattern was observed considered when solely HPV53 without any of the above mentioned HPV types except $6,11,42,43$ or 44 was detected. No additional data on potential confounding factors as HIV status and previous of current history of other sexually transmitted infections has been collected. An estimation of follow-up visit in 24 months after initial cytology and HPV genotyping result was proposed.

\section{Results}

A total of 2283 liquid-Pap samples were taken from 1465 women at Ghent University Hospital, Belgium, and from 660 women at General Hospital and Ngaliema Hospital of Kinshasa, DRC. "HPV53-only"-pattern as evaluated by full HPV genotyping was found in samples from only $34(1.6 \%)$ samples. The initial cytology of these study subjects comprised 9 ASC-US samples,
19 LSIL samples (corresponding to histological classification of cervical intraepithelial neoplasia (CIN)1), 4 HSIL samples (corresponding to histological classification of CIN2) and 2 samples revealed NILM. The data on initial cytology are presented in Table 1. HPV53 in association with any of HPV types 16, 18, 31, 33, $35,39,45,51,52,56,58,59,66,68$ was detected in $72(3.2 \%)$ of total amount of samples. For 26 (76.5\%) from the 34 women presented with "HPV53-only"-pattern follow-up results were available. The data on cytological and histological follow-up are presented in Table 2. Summarising, no evolution to high-grade lesions was found during the mean follow-up period of 26 months (range 2-48 months).

\section{Comments}

In the present study we could not find association between sole presence of HPV53 ("HPV53-only"-pattern) as investigated at the time of initial cytological examination and progression to highgrade cervical lesion over the mean follow-up period of 26 months. Our findings do not support the evidence of the carcinogenic potential of HPV53 in cervical cancer and are consistent with the previously published reports showing lack of carcinogenic activity of this pHR-HPV type [4,5,17].

The carcinogenicity of HPV types forms an evolving continuum with a permanent need of reclassification based on the newly available data. The data acquisition itself can be prone to variations based on the culturally different follow-up algorithms and endpoint evaluations, quality of cytological specimens and biopsies taken next to the variety of HPV genotyping methods available with none of them as the reference standard [18,19].

The IARC Working Group has stated in its recent report that despite being relatively commonly present in population worldwide, HPV53 occurred extremely rare alone in invasive cervical cancer and in certain geographical regions (e.g. Oceania) and noted a concern regarding inclusion of this type, HPV70 as well as HPV66 in screening assays which would decrease the specificity and positive predictive value without substantial gain in sensitivity and negative predictive value of these methods [20,2,21,22]. Especially recognising their extreme rarity in cervical cancer next to high general prevalence, studies as the current one investigating on relatively large initial cohort the progression to HSIL over time

Table 2

Follow-up cytological and histological data of "HPV 53-only" positive women.

\begin{tabular}{lll}
\hline Initial cytology & Follow-up cytology & Follow-up histology \\
\hline ASC-US (5) & NILM (3) & \\
LSIL (1) & \\
NILM (14) 16$)$ & NILM (2) \\
HSIL (4) & LSIL (1) & NILM (3) \\
NILM (1) & NILM (1)
\end{tabular}

ASC-US: atypical squamous cells of undetermined significance; (5): numbers between parenthesis refer to absolute numbers of cases with corresponding cytology or histology; NILM: negative for squamous intraepithelial lesion or malignancy; LSIL: low-grade squamous intraepithelial lesion; HSIL: high-grade squamous intraepithelial lesion. 
are of great value to gain more insight in the nature of pHR-HRV in general and HPV53 in particular.

One of the limitations of the current study is a lack of the knowledge of the HIV serological status of the study subjects. In an HIV positive population, a higher prevalence of HPV infections can be found, and often also a higher grade of co-infection with multiple HPV genotypes. Different distribution of HPV genotypes could be also noted in HIV-positive women with HSIL who were significantly more likely to be infected with HPV53 as found in the recent meta-analysis on this topic [23]. Maranga et al. describe a significantly higher prevalence of HPV53 in the HIV positive part of the study population when compared to the HIV negative controls (91.7\% of all HPV53 infections occurred in HIV positive women) [24]. However, even in the HIV positive population, there was a complete absence of HPV53 in the invasive cervical cancer cases.

An important secondary role for HPV genotypes as HPV53 could be hypothesised. Mejlhede et al. [25] suggest that infections with certain HPV types might depend on the existence of certain other HPV types. Based on this observation, a specific role for HPV53 could be suggested, inducing synergism between HPV53 and other HPV genotypes. To confirm this hypothesis, research efforts to identify commonly occurring HPV genotype pairs in multiple infections need to be performed to generate evidence for the synergistic relationship between different HPV genotypes. Important consequences would include that in the post-vaccine era, specific HPV genotypes will disappear from the population, leaving opportunity for phenomena as type replacement. It is unclear, with the selective removal of certain HPV genotypes, how the other, 'less virulent' genotypes will behave.

\section{Conflict of interest}

The authors declare that they have no conflict of interest.

\section{References}

[1] Van Ranst M, Kaplan JB, Burk RD. Phylogenetic classification of human papillomaviruses: correlation with clinical manifestations. J Gen Virol 1992;73(Pt 10):2653-60.

[2] Muñoz N, Bosch FX, de Sanjosé S, et al. Epidemiologic classification of human papillomavirus types associated with cervical cancer. N Engl J Med 2003;348: 518-27.

[3] Bouvard V, Baan R, Straif K, et al. A review of human carcinogens - Part B: biological agents. Lancet Oncol 2009;10:321-2.

[4] Meyer T, Arndt R, Beckmann ER, Padberg B, Christophers E, Stockfleth E Distribution of HPV 53, HPV 73 and CP8304 in genital epithelial lesions with different grades of dysplasia. Int J Gynecol Cancer 2001;11:198-204.

[5] Halec G, Schmitt M, Dondog B, et al. Biological activity of probable/possible high-risk human papillomavirus types in cervical cancer. Int J Cancer 2012 [Epub ahead of print].
[6] Clifford GM, Rana RK, Franceschi S, Smith JS, Gough G, Pimenta JM. Human papillomavirus genotype distribution in low-grade cervical lesions: comparison by geographic region and with cervical cancer. Cancer Epidemiol Biomarkers Prev 2005;14:1157-64.

[7] de Villiers EM, Fauquet C, Broker TR, Bernard HU, zur Hausen H. Classification of papillomaviruses. Virology 2004;324:17-27.

[8] Wyant PS, Cerqueira DM, Moraes DS, et al. Phylogeny and polymorphism in the long control region, E6, and L1 of human papillomavirus types 53, 56, and 66 in central Brazil. Int J Gynaecol Cancer 2011;21:222-9.

[9] Cento V, Rahmatalla N, Ciccozzi M, Lo Presti A, Perno CF, Ciotti M. Human papillomaviruses 53 and 66: clinical aspects and genetic analysis. Virus Res 2012;163:212-22.

[10] Oliveira LH, Santos LS, Augusto EF, Neves FP. Identification of human papillomavirus type $53 \mathrm{~L} 1, \mathrm{E} 6$ and E7 variants in isolates from Brazilian women. Infect Genet Evol 2011;12:71-6.

[11] Herrero R, Castle PE, Schiffman M, et al. Epidemiologic profile of type-specific human papillomavirus infection and cervical neoplasia in Guanacaste, Costa Rica. J Infect Dis 2005;191:1796-807.

[12] Si-Mohamed A, Ndjoyi-Mbiguino A, Cuschieri K, et al. High prevalence of highrisk oncogenic human papillomaviruses harboring atypical distribution in women of childbearing age living in Libreville, Gabon. J Med Virol 2005;77: 430-8.

[13] Oliveira LH, Ferreira MD, Augusto EF, et al. Human papillomavirus genotypes in asymptomatic young women from public schools in Rio de Janeiro, Brazil. Rev Soc Bras Med Trop 2010;43:4-8.

[14] Meyer T, Arndt R, Christophers E, et al. Association of rare human papillomavirus types with genital premalignant and malignant lesions. J Infect Dis 1998;178:252-5.

[15] Brown AJ, Trimble CL. New technologies for cervical cancer screening. Best Pract Res Clin Obstet Gynaecol 2012;26:233-42.

[16] Jeney C, Takács T, Sebe A, Schaff Z. Detection and typing of 46 genital human papillomaviruses by the L1F/L1R primer system based multiplex PCR and hybridization. J Virol Methods 2007;140:32-42.

[17] Arbyn M, Tommasino M, Depuydt C, Dillner J. Are 20 human papillomavirus types causing cervical cancer? J Pathol 2014;234:431-5.

[18] Gravitt PE, Schiffman M, Solomon D, Wheeler CM, Castle PE. A comparison of linear array and hybrid capture 2 for detection of carcinogenic human papillomavirus and cervical precancer in ASCUS-LSIL triage study. Cancer Epidemiol Biomarkers Prev 2008; 17:1248-54.

[19] Castle PE, Gravitt PE, Solomon D, Wheeler CM, Schiffman M. Comparison of linear array and line blot assay for detection of human papillomavirus and diagnosis of cervical precancer and cancer in the atypical squamous cell of undetermined significance and low-grade squamous intraepithelial lesion triage study. J Clin Microbiol 2008;46:109-17.

[20] IARC Working Group. A review of human carcinogens - Part B: Biological agents. IARC Monogr Eval Carcinog Risks Hum 2011;100B:1-475.

[21] Li N, Franceschi S, Howell-Jones R, Snijders PJ, Clifford GM. Human papillomavirus type distribution in 30,848 invasive cervical cancers worldwide: variation by geographical region, histological type and year of publication. Int J Cancer 2011;128:927-35.

[22] Bzhalava D, Guan P. Franceschi S, Dillner J, Clifford G. A systematic review of the prevalence of mucosal and cutaneous human papillomavirus types. Virology 2013;445:224-31.

[23] Clifford GM, Gonçalves MA, Franceschi S, HPV and HIV Study Group. Human papillomavirus types among women infected with HIV: a meta-analysis. AIDS 2006;20:2337-44.

[24] Maranga IO, Hampson L, Oliver AW, et al. HIV infection alters the spectrum of HPV subtypes found in cervical smears and carcinomas from Kenyan Women. Open Virol J 2013;7:19-27.

[25] Mejlhede N, Pedersen BV, Frisch M, Fomsgaard A. Multiple human papilloma virus types in cervical infections: competition or synergy? APMIS 2010;118: 346-52. 